\title{
A registration method for rigid objects without point matching
}

\author{
Yasuyo Kita \\ Electrotechnical Laboratory \\ 1-1-4 Umezono, Tsukuba-shi, Ibaraki, 305, Japan
}

\begin{abstract}
A method for the registration of rigid objects by attracting objects to the desired posture by appropriate forces is described. Inputs are a group of three-dimensional coordinates of the points representing an object in the initial state and in the goal state. No information on point correspondence between the initial and the goal states is given. Firstly, the object is translated from the initial position so that its centroid coincides with one of the goal position. The difference in posture of the object is corrected by rotating round the centroid by the torque which attracts it to the goal posture. We demonstrate that repulsive forces to each point of the object from all points at the goal posture, whose magnitude is the square of the distance between the points, satisfactorily produces such torque.
\end{abstract}

\section{Introduction}

Detecting the change in position and posture of objects which move in threedimensional (from now, abbreviated as 3D) space is one of the important subjects of computer vision. In this paper, we consider the case that an object is rigid and $3 \mathrm{D}$ data of the object is observed before and after motion of the object. The change is represented by a translation matrix and a rotation matrix which transform the initial coordinates to the final ones in the form $X^{\prime}=R * X+$ $T$. Where, $R$ is a rotation matrix, $T$ is a translation matrix, $X$ is the initial coordinates and $X^{\prime}$ is the final coordinates. As shown in some methods[1][2], $R$ and $T$ are generally calculated based on the correspondences of points or vectors (ex. normal vectors of surfaces) between the initial state and the final state. Since the number of passible correspondences of such simple elements usually becomes large, the selection of the correct pairs requires complicated processing. For example, if we observe color images with range data as shown in Fig. 1, the correspondence of the shadow region before and after the motion is relatively easy to find. However, even after that the correspondence is known, it's still difficult to find point correspondence on the contour of the region. For such cases, a method to obtain $R$ and $T$ which is not based on correspondence of points(or vectors) but based on correspondence of arbitrary units, such as contours or regions of free-form shape, is desirable.

One typical solution for this problem is based on moments[3][4]. In the method, however, not the exact but four possible rotation matrices are calculated and additional procedures are required to select the correct one from 


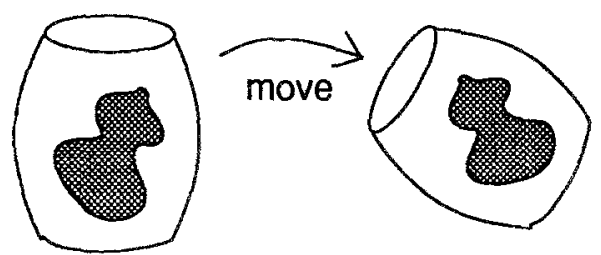

Fig. 1. Example that correspondence of points is very difficult to find, even though regions are known to correspond to each other.

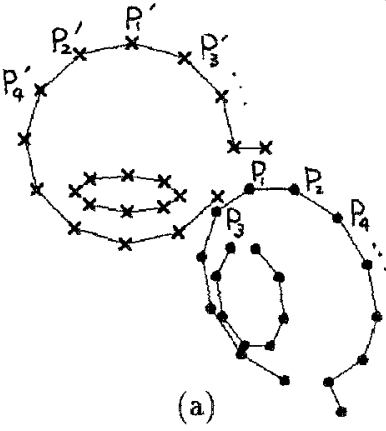

- Points at initial posture $P_{i}\left(x_{i}, y_{i}, z_{i}\right)$

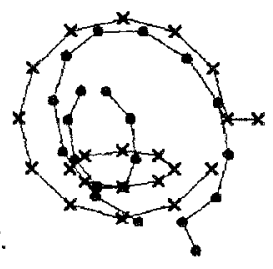

(b)

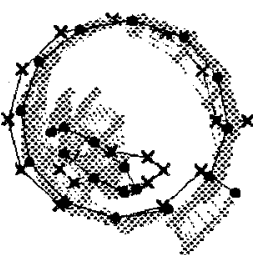

55 iterations

(c)

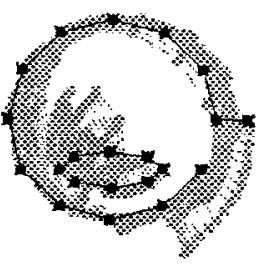

110iterations

(d)

Fig. 2. Convergence of object to goal posture

the four. Another defect in the method is that it can not detect the rotation matrix for the objects whose major and/or minor principle axis can not fixed, such as regular polyhedrons. Besides this, some other methods [5][6][7] are proposed for free-form shape matching using 3D data without the correspondence of any feature. These methods iteratively attracts observed data to model data so as to make the shortest distance between the two data as small as possible. Though these methods have advantage of allowing partial matching, they needs initial estimation of motion; if appropriate initial estimation is not given, these methods easily converge to wrong solution because of many local minima. In case that no initial estimation of rotation matrix is given, [5] needs try to start from 24 rotation matrices not to miss the global minimum.

In this paper, we propose a method to detect the rotation matrix which uses the physical rotation of the object when torque is applied to attract the object to the goal posture. Since the definition of the torque is devised not to produce many local minima, the object is stably converged to the goal posture from any posture whose angle of the rotation from the goal posture is within about $\pm \pi / 2$ radian round any axis.

\section{Outline of our method}

In our method, an object is represented as a group of points because we want to treat arbitrary shape. Figure $2 \mathrm{a}$ shows an example(3D shape by combining a large and a small circles perpendicularly): initial state is represented with $P_{i}\left(x_{i}, y_{i}, z_{i}\right)\left(\mathrm{i}=1 \sim \mathrm{n}, \bullet\right.$ in Fig. 2) and goal state is with $P_{j}^{\prime}\left(x_{j}^{\prime}, y_{j}^{\prime}, z_{j}^{\prime}\right)(\mathrm{j}=1 \sim \mathrm{n}, \times$ in 
Fig. 2). Though auxiliary lines are added for our comprehension of the 3D shape, no information is given about the structure among the points. No information is provided about correspondence of points between $P_{i}$ and $P_{j}^{\prime}$. Although the actual points and their total number are not necessarily the same before and after motion, for simplicity of explanation we assume that they are same. In subsection 3.3, we'll describe this matter in greater detail.

First, the object represented by $P_{i}$ is translated from the initial position so as to lay its centroid on that of the goal position as shown in Fig. 2b. From now, we set $P_{i}$ as the new coordinates of the object after the translation and name this state the initial posture, and $P_{j}^{\prime}$ the goal posture. Note that the object placed in the initial posture can be superposed on the goal posture if we rotate it round the centroid by applying appropriate torque to it. Therefore if we can find a definition which always gives the torque attracting the object to the goal posture in spite of how the initial posture deviates from the goal posture, the $\log$ of the rotation by the torque gives us the rotation matrix we desire.

To realize this strategy, the definition of appropriate torque is very important. A definition which quickly comes to mind might be forces such as universal gravitation. We experimentally tried such a definition using the object shown in Fig. 2. The attractive forces from all points of the goal posture are applied to each point of the object. The magnitude of the forces is defined to be inversely proportional to the square of the distance between the points (in the case where the distance $=0$, we define force $=0$ ). By rotating the object round the centroid with the torque caused by the forces, however, many local stable postures where the torque $=0$ were found besides the goal posture. Therefore, this definition is useful only when the difference between initial and goal postures is small. In the sense that this definition attracts the object mainly based on the distance between the closest points, it is similar to the other methods[5][6].

From the idea contrary to the above, a definition based on repulsive forces was experimentally tried and led to interesting results. If repulsive forces are applied to each point of the object from all points in the goal posture, whose magnitude is the square of the distance between the points, it was observed that the object is stably attracted to the goal posture when angle of the rotation from the goal posture is within about $\pm \pi / 2$ radian round any axis. In the case when the initial posture is out of the range, the object converges to an opposite posture about the centroid.

The rotation of objects is calculated in a successive approximation way ${ }^{1}$. Figure $2 \mathrm{c}$ and $2 \mathrm{~d}$ show the convergence process that $\operatorname{object}(\bullet)$ follows as it is attracted in the goal posture $(x)$. The gray lines show traces of the rotation process acting on the object. In this case, actual rotation is 90.0 degrees around the axis $(0.641,0.299,0.707)$. By our method, 90.1 degrees around the axis $(0.637,0.307,0.707)$ is obtained in a half second(SUN/SS10 workstation).

\footnotetext{
${ }^{1}$ The equation of motion is $I * d^{2} \theta / d t^{2}+\gamma d \theta / d t=N$ (Here, $I$ is the moment of inertia of the object, $\gamma$ is viscous coefficient, and $N$ is torque). If we assume that $\gamma$ is negative with a large absolute value, the acceleration(first term of the equation) can be neglected; while $d t=1$, the object rotates by $d \theta=N / \gamma$.
} 


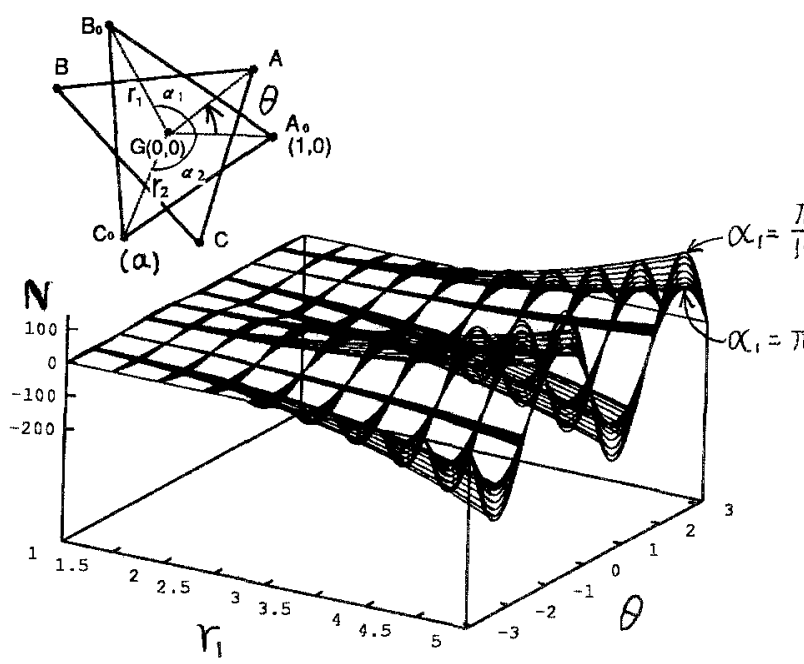

(b)

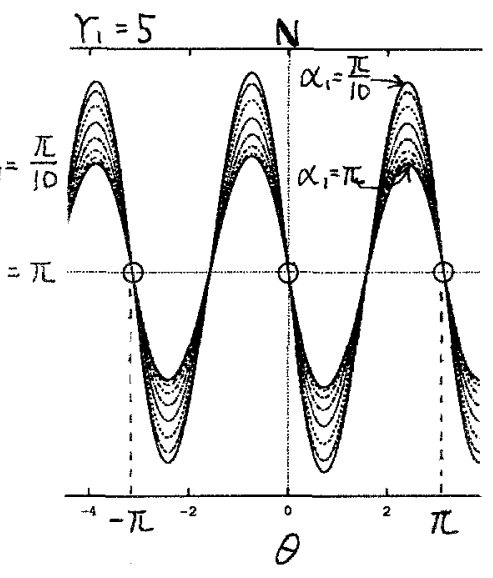

(c)

Fig. 3. Change of torque in case that objects composed of three points moves in a two dimensional polar coordinate system.

\section{Performance characteristics}

In this section, performance characteristics of the method are checked from several viewpoints.

\subsection{Characteristics of stable posture}

Let's analytically consider stable postures. Suppose that a repulsive force is applied from $P_{1}\left(r_{1}, \phi_{1}\right)$ to $P_{2}\left(r_{2}, \phi_{2}\right)$ in a polar coordinate system, whose magnitude is the square of the distance between the points. From the second cosine formula, $|\mathbf{F}|=\left|\overline{P_{1} P_{2}}\right|^{2}=r_{1}^{2}+r_{2}^{2}-2 r_{1} r_{2} \cos \theta$.

Where, $\theta=\phi_{2}-\phi_{1}$. From the sign formula, the torque caused by the force round the origin is:

$$
|\mathrm{N}|=r_{1} r_{2} \sin \theta \sqrt{r_{1}^{2}+r_{2}^{2}-2 r_{1} r_{2} \cos \theta}
$$

When the object is placed in the goal posture, repulsive forces mutually arise between any pair among all points. From the equation (1), a pair of torques between any pair of points offset each other. As a result, the goal posture is a stable posture for any object consisting of arbitrary points.

Next, let's examine how torque changes as an object shifts from the goal posture by using simple objects, which are compased of three points in a $2 \mathrm{D}$ polar coordinate system. In order to represent a triangle with few parameters, we set the three points as shown in Fig. 3a. The origin is set at the centroid of the points; two points are $\mathrm{A}(1,0)$ and $\mathrm{B}\left(r_{1}, \alpha_{1}\right)$; the remaining point $\mathrm{C}\left(r_{2}, \alpha_{2}\right)$ is determined from these conditions. That is, the parameters $r_{2}$ and $\alpha_{2}$ are a function of $r_{1}$ and $\alpha_{1}$. The torque which is produced when the object is rotated by $\theta$ from the goal state $\left(\mathrm{A}(1,0), \mathrm{B}\left(r_{1}, \alpha_{1}\right), \mathrm{C}\left(r_{2}, \alpha_{2}\right)\right)$, is calculated using 


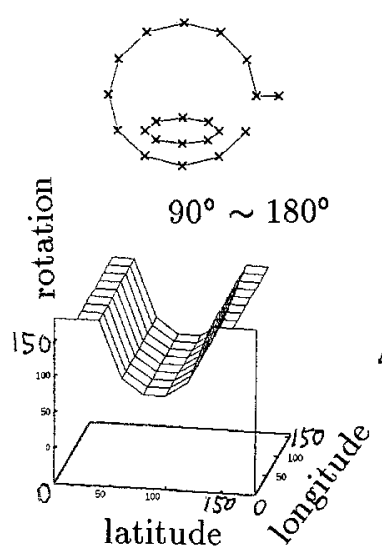

(a)

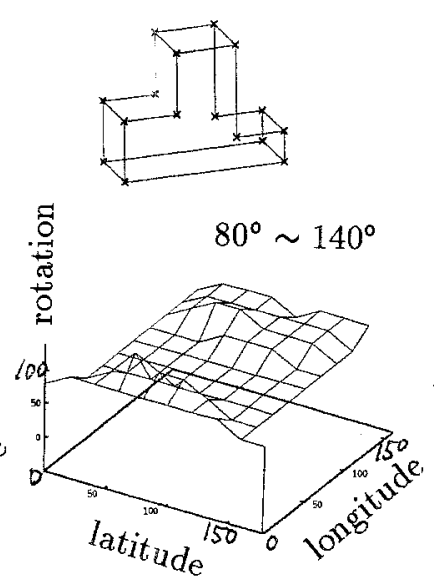

(b)

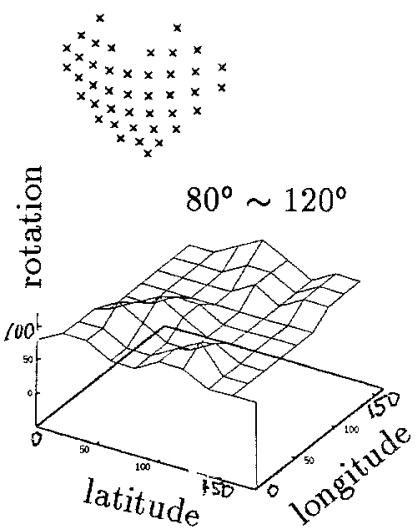

(c)

Fig. 4. Boundary angle of rotation under which objects converged to the goal posture.

the equation (1) as function of $r_{1}, \alpha_{1}$ and $\theta$. Figure $3 \mathrm{~b}$ shows the results of fixing $\alpha_{1}=\mathrm{m} \pi / 10(\mathrm{~m}=1 \sim 10)$. Figure $3 \mathrm{c}$ shows the profile at $r_{1}=5.0$. We notice that torque $=0$ at $\theta=0$ and $\pi$ and, in the near vicinity of the points, the torque has a sign opposite to the deviation from $\theta=0$ (or $\pi$ ). This brings back the object into $\theta=0$ (or $\pi$ ). As shown in the figures, these two states are the only stable states for most of triangles. Exceptions are triangles which are almost regular. In the cases of regular triangles, stable posture becomes $0,2 \pi / 3$ and $4 \pi / 3$. Objects consisting of more than four points have not yet been analytically examined.

\subsection{Experiments with various shape objects}

We applied our method to various objects. The following phenomena are observed in most of objects. When angle of the rotation from the goal posture is within about $\pm \pi / 2$ radian round an axis, objects converged to the goal posture. In the case when the initial posture is out of the range, objects can converge to a few other postures. Figure 4 shows boundary angle of rotation under which objects converged to the goal posture. In the figure, the direction of rotation axis is represented by latitude and longitude. Figure $4 \mathbf{a}$ is the boundary for the object we used in section 2 . Figure $4 \mathrm{~b}$ is one for a platform of honor represented by 14 points(vertices). Figure $4 \mathrm{c}$ is one for a heart painted on a curved surface, which is represented by 42 points selected at equal intervals.

The number of stable postures depends on the shape of objects. In the case of object in Fig. $4 \mathrm{~b}$ and $4 \mathrm{c}$, there are 4 stable postures, while the object in Fig. $4 \mathrm{a}$ has 2 stable postures. From experimental observation, the number of stable postures is expected not to become so high and is usually around 4 .

Though the moment method[4] cannot treat objects whose major and/or minor principle can not fixed (ex. regular polihedrons), our method is also useful for such objects and the object becomes stable at one of overlapping postures, which is the closest to the initial posture. 


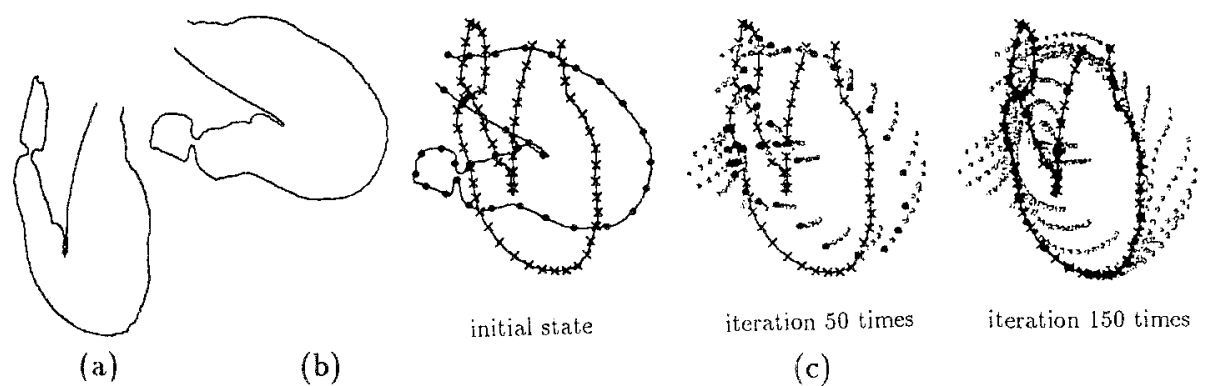

Fig. 5. Registration result in case that different representative points are selected before and after motion.

\subsection{Influence of different representative points}

Until now, we treated the same representative points before and after motion to examine the original characteristics of the attraction by the repulsive forces. Here, we show it is possible to select different representative points between the initial and goal states. Figure 5a and $5 \mathrm{~b}$ show a curve in three-dimensional before and after motion. Originally, the line is represented by 1105 points. As shown in Fig. $5 c$, representative points are selected with different regular intervals, concretely, 55 points (every 20 points) from Fig. 5 a and 33 points (every 33 points) from Fig. 5b. Figure $5 \mathrm{c}$ shows our method still can well register the object in the goal posture using different representative points. In this case, actual rotation is 60 degrees around the axis $(0.492,0.0868,-0.866)$. By our method, 59.6 degrees around the axis $(0.496,0.0867,-0.864)$ was obtained.

\subsection{Exporiment using data observed by range finder}

In order to check practicality of the method, experiments were conducted on detection of position and posture of a mug observed by a laser range finder[8]. Figure 6a shows the mug we used for the experiment. The movement of the mug from the initial position and posture is calculated based on the 3D coordinates of the contours of the solid patterns on its surface: 6 hiragana characters (" $\tau$ ", "ら", "L","や”, "す” and "よ") ${ }^{2}$. Firstly, 3D data is observed at 6 canonical states of the mug, each of which is arranged to observe each pattern. A group of $3 \mathrm{D}$ coordinates of points on the contour of each pattern are detected and memorized as a canonical pattern. For example, the second canonical pattern is represented by 3D coordinates of 38 points( $\bullet$ in Fig. 6b). For each canonical state, the positions of the hrim, the bottom and the handle of the mug are manually taught(lines in Fig. 6b).

After arbitrarily moving the mug, the new state of the mug is detected based on the pattern visible at each state. Figure $6 \mathrm{c}$ shows an example of observed $3 \mathrm{D}$

\footnotetext{
${ }^{2}$ Generally, such a pattern is easily extracted than other edges because of stable contrast with its backgronnd. In our experiments, these patterns are detected as regions that lack 3D data, since the infrared laser of the range finder is not reflected from the black patterns.
} 


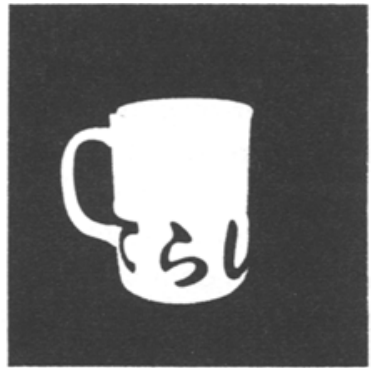

(a) mug used for experiments

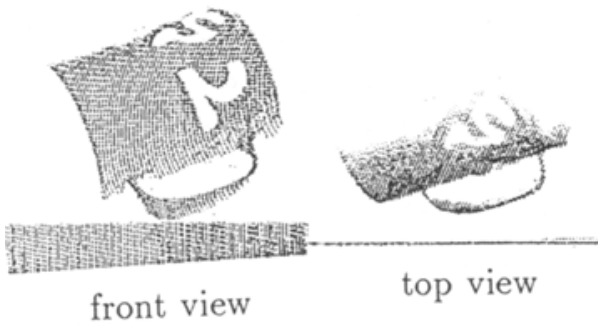

(c) observed $3 \mathrm{D}$ data

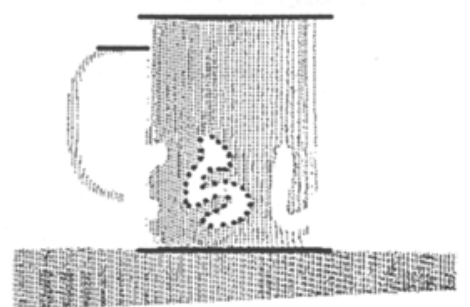

front view

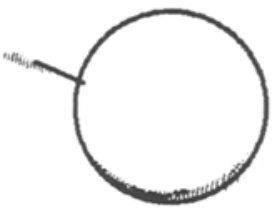

top view

(b) canonical data

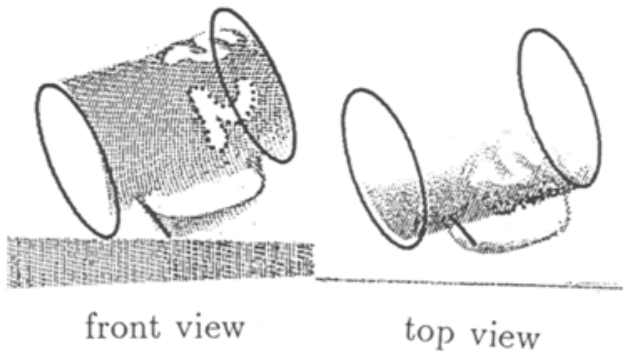

(d) results

Fig. 6. Example of detection of position and posture of mug

data. Firstly, the possible regions for a pattern are selected and a group of 3D coordinates of points on the contour of each region are detected. By using the group as points representing the goal state, each canonical pattern is registered by the proposed method. The translation matrix and rotation matrix for the best matched pattern is selected for the movement of the mug. In case of Fig. $6 c$, pattern " $\tau "$ was selected. In Fig. 6d, canonical 3D data transformed by the matrices are superposed on the observed data. It shows that the posture of the mug is well detected. For more than 10 experiments using different postures, our method gave similar results.

\section{Conclusion}

In this paper, a new simple method for registering rigid objects was described. In our method, the change in posture of an object is detected by simulating the rotation of the object when torque is applied to it so as to attract it to the goal posture. The strategy is realized based on the interesting property that repulsive forces from the goal posture stably produce an attractive torque.

One feature of our method is that the method does not need a point matching process and can use arbitrary shape, like contours or regions, as units of correspondence. This feature is useful since detecting characteristic points and finding point matching are often very difficult even in the case when correspondence of some part is easily found. 
Even though the method uses iterative process ta simulate rotation of objects, the processing time is short since one iteration is very simple and quick. The time increases proportionally to the square of the number of points representing objects and to the degree how much the initial posture shifts from the goal posture since the rotation is now simulated by unit degree( 1 degree). When an object is represented by 20 points with 50 degrees shift from the goal posture, the object converges to the goal posture in a quarter second on a SUN/SS10 workstation. The computational time can be reduced, if we devise the numerical method to simulate the converging process. Though the time increases proportionally to the square of the number of points representing objects, our methods does not need dense points to get proper registration of objects as we see in section 3.3 .

Additionally considering that the object converges to the goal posture when the shift from the goal posture is within about $\pm \pi / 2$ radian round any axis, the method is expected to be useful for tracking an object which rotates quickly.

Acknowledgements The author wishes to thank Dr. K. Fujimura for important advice, and Dr. K. Takase, Dr. F. Tomita and her colleagues of the Computer Vision Section, ETL for helpful discussions. For obtaining 3D data by the range finder, she is thankful to Mr. T. Yoshimi, Mr. T. Ueshiba and Mr. Y. Kawai. She is thankful to Dr. A. Zelinsky for his comments on English. She is grateful to Mr. N. Kita for all his help, especially for his effective advice.

\section{References}

1. O. D. Faugeras and M. Hebert: "The representation, recognition, and locating of $3 \mathrm{D}$ objects", Int. J. Robotics Research, Vol. 5, No. 3, pp. 27-52, 1986.

2. S. Umeyama, T. Kazvand and M. Hospital: "Recognition and positioning of threedimensional objects by combining matchings of primitive local patterns" , Computer Vision, Graphics 8 Image Processing, Vol. 44, No. 1, pp.58-76, 1988.

3. L. G. Brown: "A survey of image registration techniques" , ACM Computing Surveys, Vol 24, No. 4, pp.325-376, 1992.

4. J. M. Galves and M. Canton: "Normalization and shape recognition of threedimensional objects by 3D moments", Pattern Recognition, Vol. 26, No. 5, pp. $667-681,1993$.

5. P. J. Besl and N. D. Mckay: "A method for registration of 3D shapes", IEEE Trans. on Pattern Analysis and Machine Intelligence, PAMI-14, No.2, pp. 239-256, 1992.

6. L. Bruine, S. Lavallée, and R. Szeliski: "Using force fields derived from 3D distance maps for inferring the attitude of a $3 \mathrm{D}$ rigid object" "In Proc. of European Conference on Computer Vision '92, pp.670-675, 1992.

7. T. Masuda and N. Yokoya: "Robust estimation of rigid motion parameters between a pair of range images", In Proc. of The 8th Scandinavian Conference on Image Analysis, pp. 499-506, 1993.

8. T. Yoshimi and M. Oshima: "Multi light sources range finder system", In Proc. of IAPR workshop on CV, pp. 245-248, 1988. 